\title{
Lipid Droplet Formation and Lipophagy in Fatty Liver Disease
}

\author{
Ryan J. Schulze, $\mathrm{PhD}^{1} \quad$ Mark A. McNiven, $\mathrm{PhD}^{1}$ \\ ${ }^{1}$ Department of Biochemistry and Molecular Biology and the Center \\ for Digestive Diseases, Mayo Clinic, Rochester, Minnesota \\ Semin Liver Dis 2019;39:283-290.

\begin{abstract}
Address for correspondence Ryan J. Schulze, PhD, Department of Biochemistry and Molecular Biology, Mayo Clinic, 200 1st St SW, Guggenheim 16, Rochester, MN 55905

(e-mail: Schulze.Ryan@mayo.edu).
\end{abstract}

\begin{abstract}
Keywords

- autophagy

- NAFLD

- lipid droplet

Lipid droplets (LDs) are key sites of neutral lipid storage that can be found in all cells. Metabolic imbalances between the synthesis and degradation of LDs can result in the accumulation of significant amounts of lipid deposition, a characteristic feature of hepatocytes in patients with fatty liver disease, a leading indication for liver transplant in the United States. In this review, the authors highlight new literature related to the synthesis and autophagic catabolism of LDs, discussing key proteins and machinery involved in these processes. They also discuss recent findings that have revealed novel genetic risk factors associated with LD biology that contribute to lipid retention in the diseased liver.
\end{abstract}

The leading cause of chronic liver disease in the United States and Europe is nonalcoholic fatty liver disease (NAFLD), a complex manifestation of pathologies that exist on a spectrum ranging from simple steatosis (fat accumulation) to nonalcoholic steatohepatitis (NASH), fibrosis, and cirrhosis. ${ }^{1}$ Untreated, NAFLD represents a significant risk factor for the development of other metabolic disorders, including Type 2 diabetes and cardiovascular disease. Globally, NAFLD is estimated to affect nearly one in four adults and represents the second leading indication for liver transplant in the United States, ${ }^{2}$ whereas NASH is predicted to become the primary justification for transplant by $2020 .^{3}$ Furthermore, NAFLD is increasingly being recognized as a risk factor for the onset of hepatocellular carcinoma (HCC), with various studies reporting that HCC incidence rates of 4 to $22 \%$ may be directly attributable to NAFLD. ${ }^{4,5}$ Alcoholic liver disease represents a related disease state characterized by fatty liver at early stages (alcoholic steatosis) with the potential for the onset of severe complications, including alcoholic hepatitis, cirrhosis, and HCC. ${ }^{6,7}$

A defining pathophysiological hallmark of the steatotic liver is the accumulation of significant amounts of fat within the parenchymal cells of the liver, the hepatocytes. Normally, these cells are especially adept at the routine storage and efflux of fat to facilitate organismal metabolic requirements. However, in the disease state, they are susceptible to the accumulation of supraphysiological levels of fat. There is considerable evidence that abnormal levels of free fatty acids (FFAs) act as a driving force behind the pathogenesis of the more serious sequelae associated with NAFLD (i.e., NASH). As no approved pharmacological compounds exist for the treatment of the fatty liver, there remains a great deal of interest in understanding the complex relationship between fat accumulation and its effect on hepatic function.

Fatty acids (FAs) derived from peripheral storage in the adipose tissue as well as those synthesized de novo in response to carbohydrate metabolism are the two major pools that flux through the liver. In response to defects in FA oxidation or aberrant accumulation, the hepatocyte can prevent lipotoxic damage by compartmentalizing excessive FAs and sequestering them away from the cytoplasm. In hepatocytes (and indeed, most mammalian cells), the primary reservoir of fat is within a dedicated storage organelle, the cytoplasmic lipid droplet (LD). Initially considered inert depots of fat, LDs have over the course of the past three decades become appreciated to have a unique biology and are now considered to be bona fide cellular organelles. As the presence of LDs is intimately linked with the metabolic state of the hepatocyte, their biosynthesis and turnover are tightly regulated. A better understanding of the regulatory processes governing the formation and breakdown of these organelles will help guide the development of novel intervention strategies for the treatment of NAFLD. Recent 
insights into the biology of LDs have uncovered key new players governing the cellular manufacture of LDs as well as important roles for the process of autophagy, a lysosomedirected catabolic pathway of cellular self-renewal, in the turnover of LDs. In this review, we will discuss the biogenesis of LDs as well as mechanisms of autophagic turnover within the context of the fatty liver.

\section{Structure and Biogenesis of Lipid Droplets}

The LD has a unique architecture befitting a dedicated lipidstorage organelle. Generally, LDs are simple structures surrounded by an endoplasmic reticulum (ER)-derived phospholipid monolayer and decorated by a limited but dynamically changing proteome. ${ }^{8}$ The proteins on the surface of the LD are thus essential for coordination with various cytoplasmic machineries to promote further expansions in the size of the droplet, or as detailed below, the catabolism of the droplet under appropriate conditions. Within the interior of the hepatocellular LD is a neutral lipid core consisting primarily of triacylglycerol (TAG) and cholesteryl ester (CE) or retinyl ester (RE). These lipid species are each synthesized by dedicated ERlocalized acyltransferases-attractive targets for interfering with aberrant lipid accumulation in fatty liver disease.

\section{Neutral Lipid Biosynthesis}

The terminal step of triglyceride biosynthesis is catalyzed by an esterification reaction between fatty acyl-CoA and diacylglycerol by two acyl-CoA:diacylglycerol acyltransferases (DGAT1 and DGAT2). Evolutionarily distinct, these enzymes catalyze the same reaction but are thought to play differing contributions to the biogenesis of LDs. DGAT1 appears to be responsible for only a minor contribution to LD biogenesis, perhaps playing a more important role in the repackaging of excess FFAs in an effort to thwart lipotoxicity and possibly a larger role in intestinal TAG synthesis. ${ }^{9-11}$ An examination of DGAT1 null mice found decreases in adipose tissue levels, increased insulin sensitivity, and resistance to diet-induced hepatic steatosis. ${ }^{12,13}$ These promising findings resulted in the subsequent development of DGAT1-targeted pharmacological inhibitors for use in humans; however, owing to the onset of intolerable side effects, these treatments are currently thought to be of questionable utility. ${ }^{14}$ Mutations in DGAT1 have since been identified in humans that result in reduced protein levels and are correlated with congenital diarrhea, suggesting that alterations in the function of DGAT1 result in much different phenotypes between mice and humans. ${ }^{10,15}$ The second isoform, DGAT2, is highly expressed in the liver and is thought to be the predominant isoform with regard to storage of triglycerides. Owing to a unique membrane topology that is very different from DGAT1, this isoform is able to simultaneously associate with both the ER and the LD. ${ }^{16}$ Whole-animal knockouts of DGAT2 are lipopenic and die shortly following birth, likely due to insufficient triglyceride stores. ${ }^{17}$ Studies in mouse models of obesity have found that reductions in DGAT2 expression by antisense oligonucleotide treatment result in improvements to liver steatosis but also exacerbate the onset of hepatic fibrosis. ${ }^{18,19}$ Like studies with DGAT1, however, the translatability of DGAT2 inhibition from rodent studies to higher mammals has had inconclusive results, with a recent study in nonhuman primates showing that acute or chronic DGAT2 inhibition had no significant effect on triglyceride metabolism. ${ }^{20}$ Clearly, more work will be required to understand how each of these enzymes central to LD biogenesis cooperates to promote hepatic TAG synthesis and how they might be targeted to stem lipid accumulation in NAFLD.

Cholesteryl esters represent a second important type of neutral lipid stored within LDs. The acyl-CoA:cholesterol acyltransferases (ACAT1 and ACAT2) catalyze the conversion of free cholesterol to $\mathrm{CE}$, and are central to cellular cholesterol metabolism. These two enzymes are currently being investigated as drug targets for the treatment of dyslipidemias. ${ }^{21,22}$ Both isoforms are expressed in the liver, with ACAT1 showing distribution in other tissues and ACAT2 possibly being more specific to the liver. Inhibition of ACAT2 was shown to prevent dietary cholesterol-associated hepatic steatosis by promoting TAG hydrolysis and mobilization from LDs into nascent verylow-density lipoprotein particles for secretion. ${ }^{23}$ REs are a third species of neutral lipid commonly localized in LDs of the liver; however, these are found almost exclusively within hepatic stellate cells (HSCs), where they serve as the major storage form of vitamin $A .{ }^{24}$ The synthesis of RE is mediated by lecithin:retinol acyltransferase, an enzyme that appears to be essential for LD formation in HSCs. In response to liver injury, these RE-enriched LDs are rapidly mobilized and reduced in size as HSCs are activated; as a consequence, fibrosis is enhanced. ${ }^{25,26}$ How these stored REs contribute to the activation of HSCs remains unclear.

\section{Lipid Droplet Assembly}

Irrespective of the neutral lipid species generated within the ER lumen, its gradual accumulation is thought to result in a distention of the bilayer as an oil 'lens' begins to form ( - Fig. 1), with the two phospholipid leaflets of the bilayer eventually separating from each other at sites where LDs are formed. ${ }^{27,28}$ Live-cell imaging experiments demonstrated that these nascent sites of LD biogenesis are highly mobile within the ER network but can be stabilized at fixed contact sites delimited by the protein BSCL2/seipin. ${ }^{29-31}$ Seipin can therefore be considered a key governor of the initial flux of neutral lipid into the maturing LD. It may accomplish this function partly by assisting in the destabilization of the ER bilayer to permit the formation of the oil lens in the first place. ${ }^{28}$ Continued funneling of lipid into the stabilized droplet results in a directional 'budding' of the LD outward into the cytoplasm. ${ }^{32}$ Like seipin, FIT2 is a second protein that may participate in the orchestration of these initial steps in LD biogenesis, by promoting this vectorial budding process. ${ }^{27}$

Through unknown mechanisms, the LD remains either stably associated with the ER or is released freely into the cytoplasm. It remains unclear exactly at which point in this process that LD-specific proteins (e.g., the PLIN family of proteins) begin to accumulate on the growing droplet. As these early ER-LD contact sites represent critical settings for 


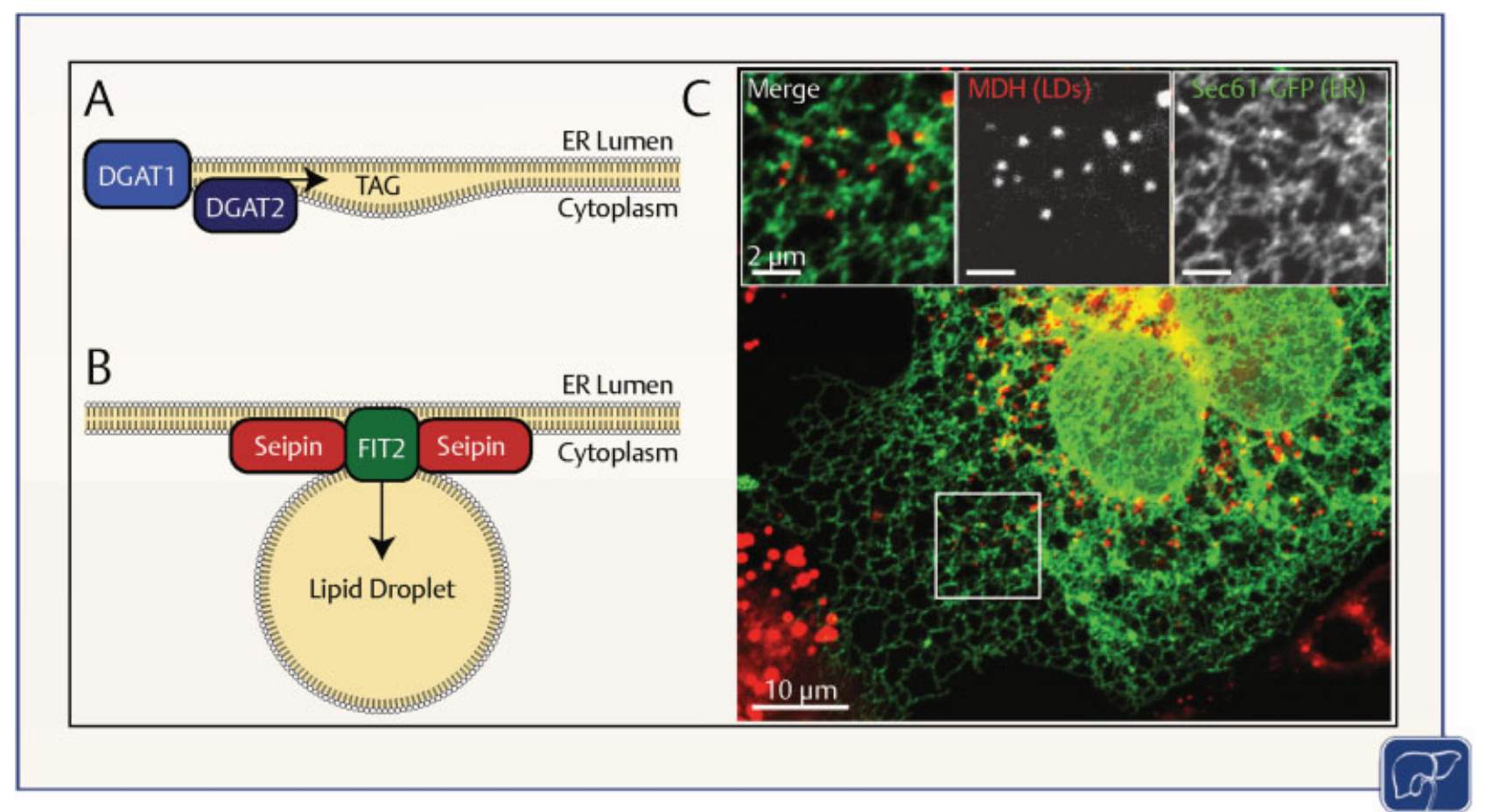

Fig. 1 Biogenesis of LDs. (A) The accumulation of neutral lipid (i.e., triglyceride synthesis by DGAT1 or DGAT2) within the ER results in a cytoplasmic distention of the outer leaflet of the ER bilayer and subsequent formation of an oil 'lens.' (B) These nascent sites of LD biogenesis can be stabilized at fixed LD-ER contact sites delimited by the proteins BSCL2/seipin and FIT2, which govern the vectorial flux of neutral lipid into the maturing LD. (C) Fluorescence microscopy of nascent LD biosynthesis as visualized by expression of Sec61-GFP (to label the ER network, green) and staining with a LD-specific dye (monodansylpentane [MDH], labeled in red). LD, lipid droplet; ER, endoplasmic reticulum.

the initial expansion of LDs and hepatic TAG accumulation, identification of the machinery involved at these cellular locations will be critical for understanding how liver steatosis might be prevented at early stages.

\section{Catabolic Pathways Mediating Lipid Droplet Breakdown}

In contrast to enhanced synthesis, decreased catabolism of LDs is another potential root cause of hepatic steatosis. Two major pathways exist for the turnover of LDs in the hepatocyte: conventional lipolysis and autophagy. Lipolysis involves a cytoplasmic cascade of enzymes (of which some directly interact with the LD) that sequentially remove one FA at a time from the glycerol backbone of the TAG stored inside the LD. Much of our understanding regarding lipolysis has been defined in adipocytes and involves three key lipases, adipose triglyceride lipase (ATGL), hormone-sensitive lipase, and monoacylglycerol lipase, which catalyze the rapid lipolysis central to adipose tissue biology. These same proteins also play important roles in the liver. The regulation of these enzymes is complex and requires the participation of many hormones and growth factors across numerous signal transduction pathways. ${ }^{33}$ For recent reviews on lipolysis, please refer to Duncan et al and Zechner et al. ${ }^{34-36}$

In addition to the soluble lipases described above, hepatocytes also utilize the autophagic pathway to catabolize LDs. This lysosome-centric process involves the construction of a specialized membrane (known as a phagophore) around the perimeter of organelles destined for degradation. Once enclosed within this autophagic membrane, in a structure referred to as an 'autophagosome,' the downstream recruitment of lysosomes to the autophagosome results in cargo degradation within a hybrid compartment known as an autolysosome ( - Fig. 2). Autophagy requires the coordinated activity of nearly 30 proteins (Atg cascade) and can be either nonselective (bulk autophagy) or selective for particular organelles such as LDs, a process referred to as "lipophagy. ${ }^{37,38} \mathrm{~A}$ seminal study by Singh and colleagues found that lipophagy plays an important role in hepatic lipid metabolism ${ }^{39}$ and that the genetic or pharmacological inhibition of autophagic flux significantly increased TAG and LD content, resulting in a concomitant decrease in hepatic FA oxidation.

Recent progress has been made toward understanding the mechanisms underlying the process of selective autophagy for other organelles such as the mitochondrion (mitophagy) or peroxisome (pexophagy) and has resulted in the identification of organelle-specific receptors that guide the assembly of the phagophore around these organelles. ${ }^{40-43}$ These receptors often contain key motifs which mediate the interaction with LC3, a protein found on the phagophore and autophagosomal membranes. ${ }^{44}$ Although no specific receptor has been identified as being present on the LD, clues from proteomic analyses have provided insights into proteins that permit lipophagy to proceed.

Because the autophagic process utilizes select components of the endocytic and lysosomal pathways, it is not surprising that a significant number of membrane trafficking proteins have been identified on the surface of the LD. While 


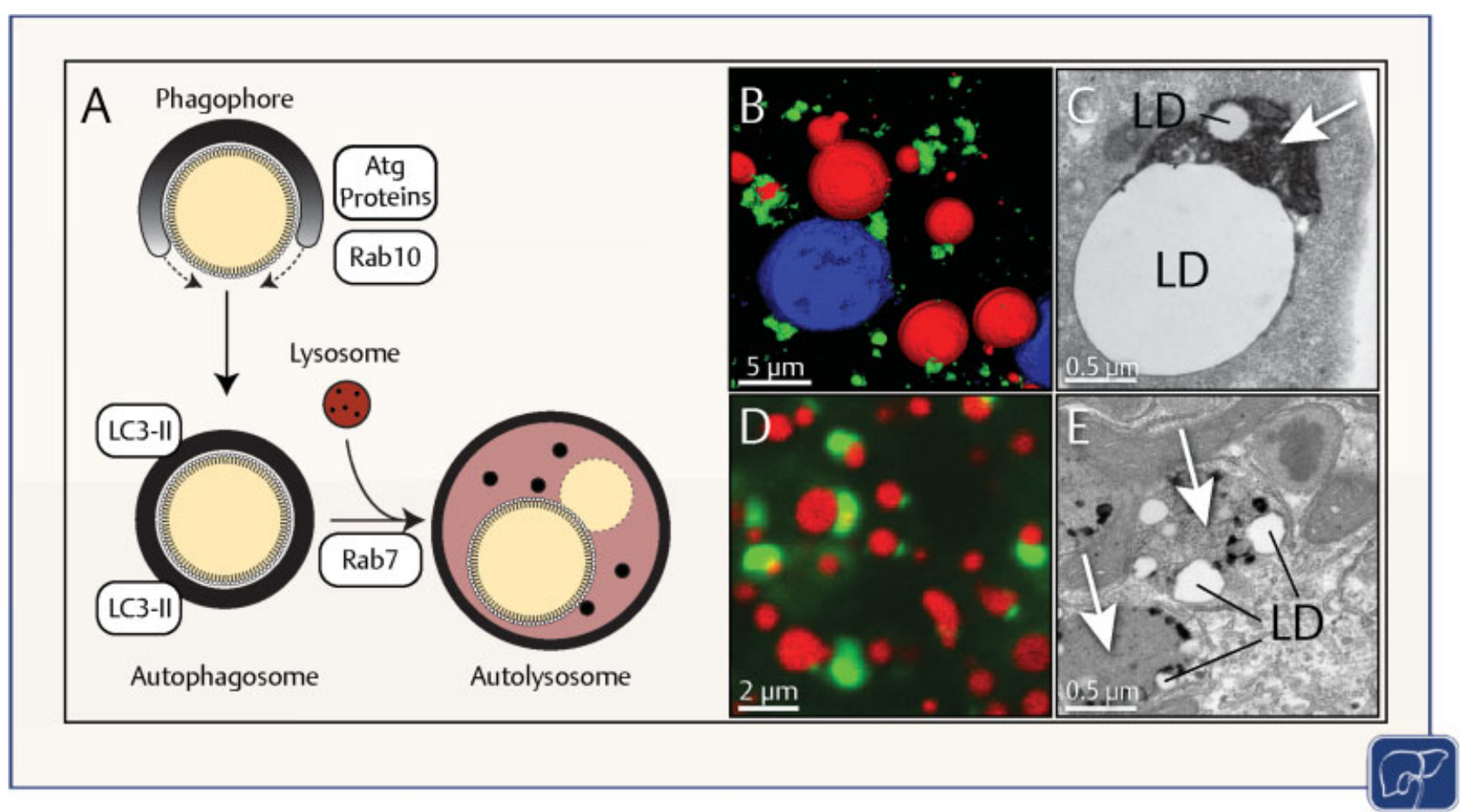

Fig. 2 Catabolism of LDs by lipophagy. (A) The process of lipophagy involves the construction of a specialized membrane (known as a phagophore) around the perimeter of LDs destined for degradation. Once enclosed within this autophagic membrane, in a structure referred to as an 'autophagosome,' the downstream recruitment of lysosomes to the autophagosome results in turnover of LDs within a hybrid compartment known as an autolysosome. (B) Maximum-intensity projection of Oil Red O-stained LDs (red) targeted by LC3-positive autophagic membranes (green) in primary porcine hepatocytes. (C) Electron micrograph of a large LD in close apposition to an autolysosome or lysosome (arrow) containing an internalized LD in HuH-7 human hepatoma cells. ${ }^{50}$ (D) Fluorescence microscopy reveals the association of a constitutively active GFP-Rab10 with LDs (red) in HuH-7 human hepatoma cells cultured in serum-starvation conditions. ${ }^{50}$ (E) Electron micrograph of a primary rat hepatocyte cultured under basal conditions and exhibiting autolysosomal structures (arrows) containing numerous enclosed LDs (these images are provided courtesy of Eugene W. Krueger, Mayo Clinic). LD, lipid droplet.

as many as 30 members of the Rab family of small GTPases have been identified to associate with LDs, the functions of just a few of these have been resolved. ${ }^{45-47}$ Among these small GTPases, Rab7 has been shown to be activated in response to nutrient depletion and to promote the association of LDs with components of the late endocytic pathway (i.e., multivesicular bodies and late endosomes/lysosomes). ${ }^{48}$ In agreement with a potential role in mediating lipophagy, inhibition of Rab7 activation results in an impairment in the turnover of hepatic LDs. ${ }^{48,49}$ Rab7 was recently shown to be a target of alcohol-induced steatosis, with activity found to be dramatically reduced in rats chronically fed an ethanol-containing diet. ${ }^{49}$ An additional Rab GTPase, Rab10, was also shown to be activated under autophagystimulating conditions and to participate in the physical engulfment of LDs by the expanding phagophore. ${ }^{50}$ Rab32, a mitochondrial protein with reported LD localization, has been shown to play a key role in LD storage in larval adipocytes from flies, with impaired autophagy upon genetic depletion. ${ }^{51,52}$ A similar role for Rab32 in LD metabolism was observed in a subsequent study. ${ }^{53}$ Another Rab GTPase, Rab18, is thought to be localized primarily to the LD as well as the ER. ${ }^{54-56}$ Although this localization suggests a direct role for this GTPase in LD catabolism, recent studies demonstrated that Rab18 depletion has no effect in several mammalian nonadipose cell types, ${ }^{57,58}$ indicating a potential cell-type specific role for this particular Rab. Further work is clearly required to dissect the many roles for these small GTPases in LD biology. In addition, large GTPases may also play critical roles in the autophagic turnover of LDs. Following degradation of LDs in the autolysosome, the autolysosomal membrane is recycled to generate nascent lysosomes in a process termed autophagic lysosome reformation (ALR). ${ }^{59}$ Long-membrane tubules are extruded from the autolysosomal membrane and undergo scission by the large GTPase dynamin-2 to regenerate lysosomes and maintain lipophagic flux. ${ }^{60}$ In the absence of dynamin-2, lengthy ALR tubules are observed in hepatocytes and LDs accumulate due to a lack of lipophagy.

Despite the differences in the mechanisms employed by the lipolytic and lipophagic pathways, there is likely to be significant crosstalk between these two processes. ${ }^{36,39,61,62}$ It was recently demonstrated that PLIN2, a major surface protein on hepatic LDs, may be selectively recognized by the chaperonemediated autophagy (CMA) system for targeted turnover. ${ }^{63}$ CMA is a quality-control process whereby proteins (such as PLIN2) harboring a specific chaperone (Hsc70)-recognition motif can be selectively imported directly into the lysosome for turnover. ${ }^{64}$ This therefore may represent a key first step in initiating downstream mechanisms of LD turnover. Removal of LD coat proteins like PLIN2 by CMA might allow for enhanced access by cytoplasmic lipases such as ATGL to act on the droplet, 
where release of FFAs could be a prerequisite for the onset of macroautophagy. A recent study provided strong evidence showing that ATGL activity was required for the downstream expression of hepatic autophagy gene expression. ${ }^{61}$ Importantly, this lipase activity also positively regulated flux through the autophagic pathway and promoted associations between the LD and components of the autophagic pathway, namely lysosomes and LC3. The pharmacological or genetic inhibition of conventional macroautophagy or lysosome-specific lipid hydrolysis was able to negate the effects of adenoviralmediated overexpression of ATGL in the hepatocyte. This research provides further evidence that ATGL action may be crucial for initiating the autophagic cascade but that lipophagy plays a more significant role than previously appreciated in the lipid turnover occurring in the hepatocyte. Other factors, such as the diameter or lipid composition of individual LDs, may also dictate the specific type of machinery used for LD catabolism.

\section{Roles for Lipophagy in Fatty Liver Disease}

Several studies have begun to address the role played by autophagy in the development of fatty liver. Insulin resistance associated with fatty liver is likely to result in the accumulation of serum FFAs available to be taken up by the liver, but the exact mechanisms leading to the accumulation of significant quantities of LDs within the fatty liver are not well understood. ${ }^{65}$ Whether the autophagic defects associated with NAFLD are a result of the excessive lipid accumulation or are themselves the actual cause is still unclear. ${ }^{65-67}$ Recent studies have examined links between autophagic defects and liver disease, although only a few have actually looked at the effects of NAFLD on the selective targeting of LDs. The transcriptional regulator transcription factor EB (TFEB), which integrates signaling and lipid homeostasis through PPAR $\alpha$ and PGC- $1 \alpha$, may itself be adversely affected in NAFLD, as mice with liver-specific TFEB knockout have significant accumulations of hepatic LDs while viralmediated hepatic overexpression of TFEB was sufficient for preventing weight gain and metabolic syndrome in mice. ${ }^{68}$ Adenoviral overexpression of TFEB in mice fed a high-fat diet interfered with the oscillating nature of an mTORC1-TFEB regulatory circuit and was able to significantly improve liver function, likely due to enhanced lysosomal function during lipophagy. ${ }^{69}$ Another possibility is that NAFLD patients may have reduced levels of glycine N-methyltransferase, an enzyme that participates in methionine and S-adenosylmethionine metabolism. ${ }^{70}$ Defects in expression of this enzyme result in elevated levels of both serum methionine and S-adenosylmethionine and consequently negatively impact hepatic lipophagy. ${ }^{70}$ Patients with NAFLD were also shown to have slightly elevated levels of rubicon, a protein with known antiautophagic properties. ${ }^{71}$ Electron microscopy performed on hepatocytes of rubicon-knockout mice showed defective hepatic lipophagy, manifested as an accumulation of double-membrane structures around the surface of numerous LDs. Mice with a liver-specific defect in macroautophagy (L-Atg5 knockout) were deficient in their adaptation to fasting-induced hepatic steatosis-the resulting liver injury and activation of nuclear factor (erythroid-derived 2)- like 2 (Nrf2) may be responsible for decreased accumulation of LDs in autophagy-deficient livers in response to fasting. ${ }^{72}$ There is also emerging evidence to suggest that hepatic cholesterol and bile acid metabolism may be linked to hepatocellular autophagic activity, with interactions potentially involved in the pathogenesis of fatty liver disease. ${ }^{73}$

Together, these studies all point to defects in hepatic lipophagy as a critical factor for the onset of NAFLD; therefore, the targeted upregulation of lipophagy may represent a viable therapeutic opportunity to promoting the resolution of fatty liver. Indeed, several autophagy inducers are under investigation as potential treatments for NAFLD. ${ }^{74}$ In one study, mice administered rapamycin exhibited an alleviation in both diet- and alcohol-induced hepatic steatosis. ${ }^{75}$ Other small molecules with potential proautophagy characteristics have also been shown to have positive effects on liver steatosis. Compounds such as celecoxib were found to alleviate NAFLD by restoring autophagic flux both in vitro and in vivo. ${ }^{76}$ Administration of minerals such as zinc, with known proautophagy properties, has been shown to promote hepatic lipophagy and reduce liver steatosis. ${ }^{77}$ Even alcohol, at least when administered acutely, appears to upregulate lipophagy in both livers of mice as well as cultured hepatocyte cell models, likely as a cytoprotective mechanism geared toward the removal of accumulated LDs. ${ }^{78,79}$

Other aspects related to the progression of fatty liver disease may also be aided by a better understanding of lipophagy. For example, it was recently shown that the vitamin A-rich LDs found in HSCs are largely catabolized in an autophagic process during HSC activation, ultimately resulting in the onset of fibrosis in NASH and cirrhosis. As HSCs are activated, autophagic flux appears to be elevated and blocking this flux in vivo has pronounced effects on liver fibrosis. ${ }^{80,81}$ Therefore, an understanding of the role for lipophagy at multiple aspects of NAFLD progression will be important.

\section{Genetic Risks for NAFLD and Connections to LDs}

A promising link to factors predisposing an individual to the onset of NAFLD was recently identified to be a regulator of hepatocellular LD metabolism. A single-nucleotide polymorphism (I148M) in a gene encoding a putative LD lipase, PNPLA3, was shown to accumulate on the surface of LDs, perhaps impeding the accessibility of the droplet to normal cytoplasmic regulatory proteins. ${ }^{82-84}$ This accumulation appears to be the result of an inherent resistance of PNPLA3 I148M to ubiquitination, suggesting that it evades normal proteasomal degradation, ultimately resulting in hepatic lipid retention and steatohepatitis. ${ }^{82}$ Patients with obesity were especially susceptible to the onset of NASH if also carrying this mutation. A search for alternative genetic links to NAFLD recently identified a mutation in the gene HSD17B13, which encodes a hydroxysteroid dehydrogenase that is also localized to the surface of LDs. ${ }^{85}$ Patients with a specific truncation variant of HSD17B13 appear to be significantly less susceptible to chronic liver disease including alcoholic and nonalcoholic cirrhosis. Furthermore, this lossof-function variant appears to mitigate the negative effects characteristic of the above PNPLA3 I148M allele. 


\section{Conclusions and Future Perspectives}

Lipid droplets represent a defining feature of fatty liver disease. These unique organelles have a biology that is only now coming into full view. From a therapeutic standpoint, a better understanding of the machinery involved in the synthesis and catabolism of LDs will offer exciting new strategies for intervention in the development of complications arising from liver steatosis.

\section{Main Concepts and Learning Points}

- A defining characteristic of fatty liver disease is the abnormal accumulation of lipid droplets (LDs), bona fide cellular organelles with unique biochemical and biophysical properties.

- LDs are synthesized in the endoplasmic reticulum (ER) by resident acyltransferases and catabolized by conventional cytoplasmic lipases (lipolysis) or within the lysosome (lipophagy).

- Several recently identified genetic links to fatty liver and chronic liver disease involve LD-associated proteins, implicating the LD itself as a target for therapeutic intervention.

\section{Conflicts of Interest}

None declared.

\section{References}

1 Younossi Z, Anstee QM, Marietti M, et al. Global burden of NAFLD and NASH: trends, predictions, risk factors and prevention. Nat Rev Gastroenterol Hepatol 2018; 15(01):11-20

2 Younossi ZM, Blissett D, Blissett R, et al. The economic and clinical burden of nonalcoholic fatty liver disease in the United States and Europe. Hepatology 2016;64(05):1577-1586

3 Wong RJ, Aguilar M, Cheung R, et al. Nonalcoholic steatohepatitis is the second leading etiology of liver disease among adults awaiting liver transplantation in the United States. Gastroenterology 2015;148(03):547-555

4 Singh S, Singh PP, Roberts LR, Sanchez W. Chemopreventive strategies in hepatocellular carcinoma. Nat Rev Gastroenterol Hepatol 2014;11(01):45-54

5 Michelotti GA, Machado MV, Diehl AM. NAFLD, NASH and liver cancer. Nat Rev Gastroenterol Hepatol 2013;10(11): 656-665

6 Altamirano J, Bataller R. Alcoholic liver disease: pathogenesis and new targets for therapy. Nat Rev Gastroenterol Hepatol 2011;8 (09):491-501

7 Singal AK, Bataller R, Ahn J, Kamath PS, Shah VH. ACG Clinical Guideline: alcoholic liver disease. Am J Gastroenterol 2018;113 (02):175-194

8 Bersuker $\mathrm{K}$, Peterson $\mathrm{CWH}$, To $\mathrm{M}$, et al. A proximity labeling strategy provides insights into the composition and dynamics of lipid droplet proteomes. Dev Cell 2018;44(01):97.e7-112.e7

9 Listenberger LL, Han X, Lewis SE, et al. Triglyceride accumulation protects against fatty acid-induced lipotoxicity. Proc Natl Acad Sci U S A 2003;100(06):3077-3082

10 van Rijn JM, Ardy RC, Kuloğlu Z, et al. Intestinal failure and aberrant lipid metabolism in patients with DGAT1 deficiency. Gastroenterology 2018;155(01):130.e15-143.e15
11 Nguyen TB, Louie SM, Daniele JR, et al. DGAT1-dependent lipid droplet biogenesis protects mitochondrial function during starvation-induced autophagy. Dev Cell 2017;42(01):9.e5-21.e5

12 Chen HC, Smith SJ, Ladha Z, et al. Increased insulin and leptin sensitivity in mice lacking acyl CoA:diacylglycerol acyltransferase 1. J Clin Invest 2002;109(08):1049-1055

13 Smith SJ, Cases S, Jensen DR, et al. Obesity resistance and multiple mechanisms of triglyceride synthesis in mice lacking Dgat. Nat Genet 2000;25(01):87-90

14 Denison H, Nilsson C, Löfgren L, et al. Diacylglycerol acyltransferase 1 inhibition with AZD7687 alters lipid handling and hormone secretion in the gut with intolerable side effects: a randomized clinical trial. Diabetes Obes Metab 2014;16(04):334-343

15 Gluchowski NL, Chitraju C, Picoraro JA, et al. Identification and characterization of a novel DGAT1 missense mutation associated with congenital diarrhea. J Lipid Res 2017;58(06):1230-1237

16 McFie PJ, Banman SL, Stone SJ. Diacylglycerol acyltransferase-2 contains a c-terminal sequence that interacts with lipid droplets. Biochim Biophys Acta Mol Cell Biol Lipids 2018;1863(09): 1068-1081

17 Stone SJ, Myers HM, Watkins SM, et al. Lipopenia and skin barrier abnormalities in DGAT2-deficient mice. J Biol Chem 2004;279 (12):11767-11776

18 Yu XX, Murray SF, Pandey SK, et al. Antisense oligonucleotide reduction of DGAT2 expression improves hepatic steatosis and hyperlipidemia in obese mice. Hepatology 2005;42(02):362-371

19 Yamaguchi K, Yang L, McCall S, et al. Inhibiting triglyceride synthesis improves hepatic steatosis but exacerbates liver damage and fibrosis in obese mice with nonalcoholic steatohepatitis. Hepatology 2007;45(06):1366-1374

20 McLaren DG, Han S, Murphy BA, et al. DGAT2 inhibition alters aspects of triglyceride metabolism in rodents but not in nonhuman primates. Cell Metab 2018;27(06):1236-1248.e6

21 Rogers MA, Liu J, Song BL, Li BL, Chang CC, Chang TY. Acyl-CoA: cholesterol acyltransferases (ACATs/SOATs): enzymes with multiple sterols as substrates and as activators. J Steroid Biochem Mol Biol 2015;151:102-107

22 Lopez AM, Chuang JC, Posey KS, et al. PRD125, a potent and selective inhibitor of sterol $\mathrm{O}$-acyltransferase 2 markedly reduces hepatic cholesteryl ester accumulation and improves liver function in lysosomal acid lipase-deficient mice. J Pharmacol Exp Ther 2015;355(02):159-167

23 Alger HM, Brown JM, Sawyer JK, et al. Inhibition of acyl-coenzyme A:cholesterol acyltransferase 2 (ACAT2) prevents dietary cholesterol-associated steatosis by enhancing hepatic triglyceride mobilization. J Biol Chem 2010;285(19):14267-14274

24 Blaner WS, O'Byrne SM, Wongsiriroj N, et al. Hepatic stellate cell lipid droplets: a specialized lipid droplet for retinoid storage. Biochim Biophys Acta 2009;1791(06):467-473

25 Jophlin LL, Koutalos Y, Chen C, Shah V, Rockey DC. Hepatic stellate cells retain retinoid-laden lipid droplets after cellular transdifferentiation into activated myofibroblasts. Am J Physiol Gastrointest Liver Physiol 2018;315(05):G713-G721

26 Tsuchida T, Friedman SL. Mechanisms of hepatic stellate cell activation. Nat Rev Gastroenterol Hepatol 2017;14(07):397-411

27 Choudhary V, Ojha N, Golden A, Prinz WA. A conserved family of proteins facilitates nascent lipid droplet budding from the ER. J Cell Biol 2015;211(02):261-271

28 Henne WM, Reese ML, Goodman JM. The assembly of lipid droplets and their roles in challenged cells. EMBO J 2018;37 (12):37

29 Wang H, Becuwe M, Housden BE, et al. Seipin is required for converting nascent to mature lipid droplets. eLife 2016;5:5

30 Szymanski KM, Binns D, Bartz R, et al. The lipodystrophy protein seipin is found at endoplasmic reticulum lipid droplet junctions and is important for droplet morphology. Proc Natl Acad Sci U S A 2007;104(52):20890-20895 
31 Cartwright BR, Binns DD, Hilton CL, Han S, Gao Q, Goodman JM. Seipin performs dissectible functions in promoting lipid droplet biogenesis and regulating droplet morphology. Mol Biol Cell 2015;26(04):726-739

32 Chorlay A, Thiam AR. An asymmetry in monolayer tension regulates lipid droplet budding direction. Biophys J 2018;114 (03):631-640

33 Schott MB, Rasineni K, Weller SG, et al. $\beta$-Adrenergic induction of lipolysis in hepatocytes is inhibited by ethanol exposure. J Biol Chem 2017;292(28):11815-11828

34 Duncan RE, Ahmadian M, Jaworski K, Sarkadi-Nagy E, Sul HS. Regulation of lipolysis in adipocytes. Annu Rev Nutr 2007; 27:79-101

35 Zechner R, Zimmermann R, Eichmann TO, et al. FAT SIGNALSlipases and lipolysis in lipid metabolism and signaling. Cell Metab 2012;15(03):279-291

36 Zechner R, Madeo F, Kratky D. Cytosolic lipolysis and lipophagy: two sides of the same coin. Nat Rev Mol Cell Biol 2017;18(11): 671-684

37 Gatica D, Lahiri V, Klionsky DJ. Cargo recognition and degradation by selective autophagy. Nat Cell Biol 2018;20(03):233-242

38 Okamoto K. Organellophagy: eliminating cellular building blocks via selective autophagy. J Cell Biol 2014;205(04):435-445

39 Singh R, Kaushik S, Wang Y, et al. Autophagy regulates lipid metabolism. Nature 2009;458(7242):1131-1135

40 McWilliams TG, Muqit MM. PINK1 and Parkin: emerging themes in mitochondrial homeostasis. Curr Opin Cell Biol 2017; 45:83-91

41 Rodger CE, McWilliams TG, Ganley IG. Mammalian mitophagy from in vitro molecules to in vivo models. FEBS J 2018;285(07): 1185-1202

42 Nordgren M, Francisco T, Lismont C, et al. Export-deficient monoubiquitinated PEX5 triggers peroxisome removal in SV40 large T antigen-transformed mouse embryonic fibroblasts. Autophagy 2015;11(08):1326-1340

43 Zhang J, Tripathi DN, Jing J, et al. ATM functions at the peroxisome to induce pexophagy in response to ROS. Nat Cell Biol 2015;17 (10):1259-1269

44 Birgisdottir AB, Lamark T, Johansen T. The LIR motif - crucial for selective autophagy. J Cell Sci 2013;126(Pt 15):3237-3247

45 Krahmer N, Hilger M, Kory N, et al. Protein correlation profiles identify lipid droplet proteins with high confidence. Mol Cell Proteomics 2013;12(05):1115-1126

46 Rasineni K, McVicker BL, Tuma DJ, McNiven MA, Casey CA. Rab GTPases associate with isolated lipid droplets (LDs) and show altered content after ethanol administration: potential role in alcohol-impaired LD metabolism. Alcohol Clin Exp Res 2014;38 (02):327-335

47 Kiss RS, Nilsson T. Rab proteins implicated in lipid storage and mobilization. J Biomed Res 2014;28(03):169-177

48 Schroeder B, Schulze RJ, Weller SG, Sletten AC, Casey CA, McNiven MA. The small GTPase Rab7 as a central regulator of hepatocellular lipophagy. Hepatology 2015;61(06):1896-1907

49 Schulze RJ, Rasineni K, Weller SG, et al. Ethanol exposure inhibits hepatocyte lipophagy by inactivating the small guanosine triphosphatase Rab7. Hepatol Commun 2017;1(02):140-152

50 Li Z, Schulze RJ, Weller SG, et al. A novel Rab10-EHBP1-EHD2 complex essential for the autophagic engulfment of lipid droplets. Sci Adv 2016;2(12):e1601470

51 Wang C, Liu Z, Huang X. Rab32 is important for autophagy and lipid storage in drosophila. PLoS One 2012;7(02):e32086

52 Alto NM, Soderling J, Scott JD. Rab32 is an A-kinase anchoring protein and participates in mitochondrial dynamics. J Cell Biol 2002;158(04):659-668

53 Li Q, Wang J, Wan Y, Chen D. Depletion of Rab32 decreases intracellular lipid accumulation and induces lipolysis through enhancing ATGL expression in hepatocytes. Biochem Biophys Res Commun 2016;471(04):492-496
54 Martin S, Driessen K, Nixon SJ, Zerial M, Parton RG. Regulated localization of Rab18 to lipid droplets: effects of lipolytic stimulation and inhibition of lipid droplet catabolism. J Biol Chem 2005;280(51):42325-42335

55 Ozeki S, Cheng J, Tauchi-Sato K, Hatano N, Taniguchi H, FujimotoT. Rab18 localizes to lipid droplets and induces their close apposition to the endoplasmic reticulum-derived membrane. J Cell Sci 2005;118(Pt 12):2601-2611

56 Gerondopoulos A, Bastos RN, Yoshimura S, et al. Rab18 and a Rab18 GEF complex are required for normal ER structure. J Cell Biol 2014;205(05):707-720

57 Jayson CBK, Arlt H, Fischer AW, Lai ZW, Farese RV Jr, Walther TC. Rab18 is not necessary for lipid droplet biogenesis or turnover in human mammary carcinoma cells. Mol Biol Cell 2018;29(17): 2045-2054

58 Xu D, Li Y, Wu L, et al. Rab18 promotes lipid droplet (LD) growth by tethering the ER to LDs through SNARE and NRZ interactions. J Cell Biol 2018;217(03):975-995

59 Yu L, McPhee CK, Zheng L, et al. Termination of autophagy and reformation of lysosomes regulated by mTOR. Nature 2010;465 (7300):942-946

60 Schulze RJ, Weller SG, Schroeder B, et al. Lipid droplet breakdown requires dynamin 2 for vesiculation of autolysosomal tubules in hepatocytes. J Cell Biol 2013;203(02):315-326

61 Sathyanarayan A, Mashek MT, Mashek DG. ATGL promotes autophagy/lipophagy via SIRT1 to control hepatic lipid droplet catabolism. Cell Reports 2017;19(01):1-9

62 Martinez-Lopez N, Garcia-Macia M, Sahu S, et al. Autophagy in the CNS and periphery coordinate lipophagy and lipolysis in the brown adipose tissue and liver. Cell Metab 2016;23(01):113-127

63 Kaushik S, Cuervo AM. Degradation of lipid droplet-associated proteins by chaperone-mediated autophagy facilitates lipolysis. Nat Cell Biol 2015;17(06):759-770

64 Kaushik S, Cuervo AM. The coming of age of chaperone-mediated autophagy. Nat Rev Mol Cell Biol 2018;19(06):365-381

65 Czaja MJ. Function of autophagy in nonalcoholic fatty liver disease. Dig Dis Sci 2016;61(05):1304-1313

66 Kwanten WJ, Martinet W, Michielsen PP, Francque SM. Role of autophagy in the pathophysiology of nonalcoholic fatty liver disease: a controversial issue. World J Gastroenterol 2014;20 (23):7325-7338

67 Martinez-Lopez N, Singh R. Autophagy and lipid droplets in the liver. Annu Rev Nutr 2015;35:215-237

68 Settembre C, De Cegli R, Mansueto G, et al. TFEB controls cellular lipid metabolism through a starvation-induced autoregulatory loop. Nat Cell Biol 2013;15(06):647-658

69 Zhang H, Yan S, Khambu B, et al. Dynamic MTORC1-TFEB feedback signaling regulates hepatic autophagy, steatosis and liver injury in long-term nutrient oversupply. Autophagy 2018;14 (10):1779-1795

70 Zubiete-Franco I, García-Rodríguez JL, Martínez-Uña M, et al. Methionine and S-adenosylmethionine levels are critical regulators of PP2A activity modulating lipophagy during steatosis. J Hepatol 2016;64(02):409-418

71 Tanaka S, Hikita H, Tatsumi T, et al. Rubicon inhibits autophagy and accelerates hepatocyte apoptosis and lipid accumulation in nonalcoholic fatty liver disease in mice. Hepatology 2016;64(06): 1994-2014

72 Li Y, Chao X, Yang L, et al. Impaired fasting-induced adaptive lipid droplet biogenesis in liver-specific Atg5-deficient mouse liver is mediated by persistent nuclear factor-like 2 activation. Am J Pathol 2018;188(08):1833-1846

73 Wang Y, Ding WX, Li T. Cholesterol and bile acid-mediated regulation of autophagy in fatty liver diseases and atherosclerosis. Biochim Biophys Acta Mol Cell Biol Lipids 2018;1863(07):726-733

74 Ni HM, Williams JA, Yang H, Shi YH, Fan J, Ding WX. Targeting autophagy for the treatment of liver diseases. Pharmacol Res 2012;66(06):463-474 
75 Lin CW, Zhang $\mathrm{H}$, Li M, et al. Pharmacological promotion of autophagy alleviates steatosis and injury in alcoholic and nonalcoholic fatty liver conditions in mice. J Hepatol 2013;58(05): 993-999

76 Liu C, Liu L, Zhu HD, et al. Celecoxib alleviates nonalcoholic fatty liver disease by restoring autophagic flux. Sci Rep 2018;8(01):4108

77 Wei CC, Luo Z, Hogstrand C, et al. Zinc reduces hepatic lipid deposition and activates lipophagy via $\mathrm{Zn}^{2+} / \mathrm{MTF}-1 / \mathrm{PPAR} \alpha$ and $\mathrm{Ca}^{2+} /$ CaMKKß/AMPK pathways. FASEB J 2018;32(12):6666-6680

78 Wang L, Zhou J, Yan S, Lei G, Lee CH, Yin XM. Ethanol-triggered lipophagy requires SQSTM1 in AML12 hepatic cells. Sci Rep 2017; 7(01):12307

79 Ding WX, Li M, Chen X, et al. Autophagy reduces acute ethanolinduced hepatotoxicity and steatosis in mice. Gastroenterology 2010;139(05):1740-1752

80 Hernández-Gea V, Ghiassi-Nejad Z, Rozenfeld R, et al. Autophagy releases lipid that promotes fibrogenesis by activated hepatic stellate cells in mice and in human tissues. Gastroenterology 2012;142(04):938-946

81 Thoen LF, Guimarães EL, Dollé L, et al. A role for autophagy during hepatic stellate cell activation. J Hepatol 2011;55(06):1353-1360

82 BasuRay S, Smagris E, Cohen JC, Hobbs HH. The PNPLA3 variant associated with fatty liver disease (I148M) accumulates on lipid droplets by evading ubiquitylation. Hepatology 2017;66(04): 1111-1124

83 Romeo S, Kozlitina J, Xing C, et al. Genetic variation in PNPLA3 confers susceptibility to nonalcoholic fatty liver disease. Nat Genet 2008;40(12):1461-1465

84 Smagris E, BasuRay S, Li J, et al. Pnpla3I148M knockin mice accumulate PNPLA3 on lipid droplets and develop hepatic steatosis. Hepatology 2015;61(01):108-118

85 Abul-Husn NS, Cheng X, Li AH, et al. A protein-truncating HSD17B13 variant and protection from chronic liver disease. $\mathrm{N}$ Engl J Med 2018;378(12):1096-1106 